\title{
Impurity-induced spin polarization and NMR line broadening in underdoped cuprates
}

\author{
R. Kilian, S. Krivenko, G. Khaliullin $\mathrm{A}$ and P. Fulde \\ Max-Planck-Institut für Physik komplexer Systeme, Nöthnitzer Strasse 38, D-01187 Dresden, Germany
}

(Version date: May 11, 2018)

\begin{abstract}
We present a theory of magnetic $(S=1) \mathrm{Ni}$ and nonmagnetic Zn impurities in underdoped cuprates. Both types of impurities are shown to induce $S=\frac{1}{2}$ moments on $\mathrm{Cu}$ sites in the proximity of the impurity, a process which is intimately related to the spin gap phenomenon in cuprates. Below a characteristic Kondo temperature, the $\mathrm{Ni}$ spin is partially screened by the $\mathrm{Cu}$ moments, resulting in an effective impurity spin $S=\frac{1}{2}$. We further analyze the Ruderman-Kittel-Kasiya-Yosida-type response of planar $\mathrm{Cu}$ spins to a polarization of the effective impurity moments and derive expressions for the corresponding ${ }^{17} \mathrm{O}$ NMR line broadening. The peculiar aspects of recent experimental NMR data can be traced back to different spatial characteristics of $\mathrm{Ni}$ and $\mathrm{Zn}$ moments as well as to an inherent temperature dependence of local antiferromagnetic correlations.
\end{abstract}

PACS numbers: 75.20.Hr, 74.25.Ha, 74.72.-h, 76.60.-k

\section{INTRODUCTION}

The normal state of underdoped cuprates exhibits unusual magnetic properties which are believed to be intimately related to the mechanism of high- $T_{c}$ superconductivity. Most peculiar in this respect are the simultaneous occurrence of a magnetic pseudogap and the persistence of antiferromagnetic (AF) correlations as holes are doped into the antiferromagnetic insulator and the system becomes metallic. It is one of the most challenging theoretical problems in the physics of high- $T_{c}$ cuprates to reconcile the gaplike features reminiscent of a spin liquid with the presence of antiferromagnetic correlations signaling the closeness of the system to a spin-ordered Néel state. Experimentally, insight into the nature of these anomalous features can be gained by introducing impurities into the magnetically active $\mathrm{Cu}$ sites. A subsequent NMR probe on nuclei coupled to the $\mathrm{CuO}_{2}$ planes yields information on the local magnetic structure. In this paper we present a microscopic theory of the impurity-induced local spin polarization of $\mathrm{CuO}_{2}$ planes and its impact on the NMR linewidth.

Introducing magnetically active or inert impurities into underdoped cuprates leads, in both cases, to the formation of local magnetic moments. Specifically, $\mathrm{Cu}\left(d^{9}\right)$ with an effective in-plane spin $S=\frac{1}{2}$ can be replaced by Ni $\left(d^{8}\right)$ with $S=1$ or Zn $\left(d^{10}\right)$ with $S=0$. Superconducting quantum interference device (SQUID) measurements of the macroscopic susceptibilityl reveal an almost perfect $1 / T$ Curie behavior. Recently, Bobroff et al.2 presented NMR measurements on ${ }^{17} \mathrm{O}$ for the underdoped compound $\mathrm{YBa}_{2}\left(\mathrm{Cu}_{1-x} M_{x}\right)_{3} \mathrm{O}_{6.6}$, with $M=\mathrm{Zn}$ or $\mathrm{Ni}$. The polarization of $\mathrm{Cu}$ spins in the presence of impurities leads to a broadening of the NMR line. In contrast to the aforementioned SQUID measurement, the linewidth displays a marked non-Curie behavior, indicating an inherent temperature dependence of the polarizability of $\mathrm{CuO}_{2}$ planes. This was suggested by Morr et al. . $^{3}$ to be a clear indication for a temperature dependence of the $\mathrm{AF}$ correlation length. Still another interesting observation can be made by comparing the two experiments: While the NMR study shows nonmagnetic Zn to have a more pronounced effect on the linewidth than $\mathrm{Ni}$, measurements of the macroscopic susceptibility reveal a reversed effect. Since only the NMR experiment is sensitive to a spatial variation of the spin polarization, a very different shape of the spin density induced by the two types of impurities can be inferred.

In the following, we present a microscopic theory of moments induced by magnetic and nonmagnetic impurities in the spin gap phase of underdoped cuprates. We analyze the different nature of coupling between $\mathrm{Cu}$ and impurity spins and derive expressions for the local spin polarization of $\mathrm{CuO}_{2}$ planes. The presence of the spin gap and of short-range AF correlations is shown to strongly modify the conventional Ruderman-KittelKasiya-Yosida (RKKY) picture. Finally, we derive expressions for the NMR line broadening which account well for the peculiarities of the experimental data.

\section{IMPURITY MODEL}

The relevant physics of the $\mathrm{CuO}_{2}$ planes of high- $T_{c}$ cuprates is believed to be described by the large- $U$ Hubbard or $t-J$ model. The dualism between itinerant charge motion and local electron interaction that is inherent to these models can, in an approximate way, be captured by introducing separate quasiparticles for spin and charge degrees of freedom. Within this picture, the normal state of underdoped cuprates is viewed as a phase in which spins form singlet pairs while coherence between holes that would eventually lead to superconductivity has not been established. We follow this line of thinking but restrict ourselves to the magnetic sector of the Hilbert space. Our starting point is the spin- $\frac{1}{2}$ AF Heisenberg model $H_{J}=J \sum_{\langle i j\rangle} \boldsymbol{s}_{i} \boldsymbol{s}_{j}$. Keeping in mind the presence of itinerant holes which prevent the system from developing long-range magnetic order, we treat this Hamiltonian within resonance valence bond (RVB) mean-field theory - this accounts well for the spin-liquid features of cuprates. The mean-field Hamiltonian is 


$$
H_{\mathrm{RVB}}=-\sum_{\langle i j\rangle \sigma}\left(\Delta_{i j} f_{i \sigma}^{\dagger} f_{j \sigma}+\text { H.c. }\right) .
$$

Original spin operators $s_{i}$ have been expressed in terms of fermionic operators by $\boldsymbol{s}_{i}=\frac{1}{2} \sum_{\sigma \sigma^{\prime}} \boldsymbol{\tau}_{\sigma \sigma^{\prime}} f_{i \sigma}^{\dagger} f_{i \sigma^{\prime}}$ with Pauli matrix vector $\boldsymbol{\tau}=\left(\tau^{x}, \tau^{y}, \tau^{z}\right)$. The local constraint prohibiting a double occupancy of sites has been relaxed to a global one. The mean-field bond parameter is $\Delta_{i j}=\Delta_{\delta}=J \sum_{\sigma}\left\langle f_{i+\delta, \sigma}^{\dagger} f_{i \sigma}\right\rangle^{0}$, where $\langle\cdots\rangle^{0}$ is the expectation value that corresponds to Hamiltonian (1). The phase of this mean-field parameter is yet undetermined and has to be chosen such as to resemble the experimental situation most closely. An appropriate choice for the spin gap regime is the flux phase $\Delta_{ \pm x}=i \Delta_{ \pm y} \equiv \Delta$. Dividing the lattice into two sublattices $A$ and $B$ and going to the momentum representation, Hamiltonian (11) can be diagonalized

$$
H_{\mathrm{RVB}}=\sum_{\boldsymbol{k} \nu} \xi_{\boldsymbol{k}}^{\nu} f_{\boldsymbol{k} \nu}^{\dagger} f_{\boldsymbol{k} \nu}
$$

with index $\nu= \pm$. The spectrum of spin excitations or spinons is

$$
\xi_{\boldsymbol{k}}^{ \pm}= \pm 2 \Delta\left(\cos ^{2} k_{x}+\cos ^{2} k_{y}\right)^{1 / 2} .
$$

It has nodes at $( \pm \pi / 2, \pm \pi / 2)$, yielding a $\mathrm{V}$-shaped pseudogap in the density of states centered at the spinon chemical potential $\mu_{s}=0: \rho^{(0)}(\omega)=|\omega| / D^{2}$ (defined per spin up/down state), where $D=2 \sqrt{\pi} \Delta$ is the spinon half-band-width.

To simulate first a nonmagnetic Zn impurity we introduce into Hamiltonian (11) a local chemical potential $\lambda$ acting on site $\boldsymbol{R}=0$, which by convention lies on sublattice $A$. In the limit $\lambda \rightarrow \infty$ spinons are expelled from that site, creating a vacancy. The Hamiltonian is then

$$
H_{\mathrm{Zn}}=H_{\mathrm{RVB}}+\left.\lambda \sum_{\sigma} f_{0 \sigma}^{\dagger} f_{0 \sigma}\right|_{\lambda \rightarrow \infty} .
$$

To describe a magnetic Ni impurity, into the empty site we insert an impurity spin $\boldsymbol{S}_{0}$ with $S=1$ which is coupled antiferromagnetically to the surrounding $\mathrm{Cu}$ spins $\boldsymbol{s}_{\boldsymbol{\delta}}$. The corresponding Hamiltonian is

$$
H_{\mathrm{Ni}}=H_{\mathrm{RVB}}+\left.\lambda \sum_{\sigma} f_{0 \sigma}^{\dagger} f_{0 \sigma}\right|_{\lambda \rightarrow \infty}+H_{\mathrm{imp}}
$$

with the exchange interaction term

$$
H_{\mathrm{imp}}=J^{\prime} \sum_{\delta} \boldsymbol{S}_{0} \boldsymbol{s}_{\boldsymbol{\delta}} .
$$

Formally, Hamiltonian (14) differs from Eq. (3) only in the presence of an additional term $H_{\mathrm{imp}} \propto J^{\prime}$. In the following, we put emphasis on the case of a magnetic impurity with $J^{\prime}>0$. A nonmagnetic impurity can be simulated by setting $J^{\prime}=0$, which decouples the impurity site from the rest of the system. The $S=1$ impurity spin is then free and can easily be disregarded. We discuss this limit in the following, but only shortly. More detailed treatments on nonmagnetic impurities are given in Ref. 6 as well as in Refs. 아 9.

\section{LOCAL MAGNETIC MOMENTS}

We analyze an impurity spin $S=1$ embedded in a spin gap system as described by Hamiltonian (4). Spinons stemming from the initial $\mathrm{Cu}$ spin at site $\boldsymbol{R}=0$ are ejected by the local potential $\lambda$. The impurity spin, which is placed in the vacant site, is conveniently represented by two spins $\frac{1}{2}$, i.e., $\boldsymbol{S}=\boldsymbol{S}_{a}+\boldsymbol{S}_{b}$. An infinitely strong ferromagnetic interaction $H_{c}=-J_{c} \boldsymbol{S}_{a} \boldsymbol{S}_{b}$ between these two spins is assumed. Expressing $\boldsymbol{S}_{a}$ and $\boldsymbol{S}_{b}$ in terms of fermionic operators $a_{\sigma}$ and $b_{\sigma}$, respectively, a mean-field decoupling can be performed:

$$
H_{\mathrm{imp}}=-\sum_{\delta \sigma}\left(\Delta_{\delta}^{\prime} \frac{a_{\sigma}^{\dagger}+b_{\sigma}^{\dagger}}{\sqrt{2}} f_{\boldsymbol{\delta} \sigma}+\text { H.c. }\right)-J_{c} \boldsymbol{S}_{a} \boldsymbol{S}_{b} .
$$

Introducing operators $f_{0 \sigma}=\left(a_{\sigma}+b_{\sigma}\right) / \sqrt{2}$ and $d_{\sigma}=$ $\left(a_{\sigma}-b_{\sigma}\right) / \sqrt{2}$, one obtains

$$
H_{\mathrm{imp}}=-\sum_{\boldsymbol{\delta} \sigma}\left(\Delta_{\delta}^{\prime} f_{0 \sigma}^{\dagger} f_{\boldsymbol{\delta} \sigma}+\text { H.c. }\right)-J_{c} \boldsymbol{S}_{\mathrm{eff}} \boldsymbol{s}_{0} .
$$

The impurity spin has thus been decomposed into two $S=\frac{1}{2}$ effective spins $\boldsymbol{S}_{\text {eff }}$ and $\boldsymbol{s}_{0}$. The former is represented by operators $d_{\sigma}$, the latter by operators $f_{0 \sigma}$. Due to the first term in Eq. (6), the $f$ spinons on the impurity site hybridize with the ones on adjacent $\mathrm{Cu}$ sites. This process is controlled by the local mean-field parameter $\Delta_{\delta}^{\prime}=J^{\prime} \sum_{\sigma}\left\langle f_{\delta \sigma}^{\dagger} f_{0 \sigma}\right\rangle$ replacing $\Delta_{\delta}$ on bonds connecting to the impurity. A system of itinerant spinons extending over the whole lattice including the impurity site is formed. These itinerant spinons couple ferromagnetically to the localized spin $\boldsymbol{S}_{\text {eff. }}$. In the presence of a magnetic field this coupling is responsible for a polarization of the spinon system to be discussed in Sec. IV. The $T$ matrix that describes scattering of spinons on the localized spin vanishes as $T(\omega) \propto \omega \ln |\omega|$ in the flux phase 6 This means that the effective local spin $\boldsymbol{S}_{\text {eff }}$ becomes asymptotically free in the limit of low energies. In the remainder of the present section we analyze this low-energy fixed point, emphasizing the role of bond parameters $\Delta_{\delta}^{\prime}$ that induce an inhomogeneity in the spinon sector.

First we consider the special case of equal exchange integrals $J^{\prime}=J$. Regarding the spinon sector, the impurity site becomes indistinguishable from the rest of the system as $\Delta_{\delta}^{\prime}=\Delta_{\delta}$. The spin $s_{0}$ takes the role of the original $\mathrm{Cu}$ spin at $\boldsymbol{R}=0$, and a homogeneous spin liquid, as described by $H_{\mathrm{RVB}}$, Eq. (1), is formed. Generally, the two exchange integrals differ, $J^{\prime}<J$, and translational invariance of the spinon system is broken. The bond parameter then acquires an additional spatial dependence which has to be treated self-consistently. To simplify the discussion, however, we distinguish only between bonds that do and do not connect to the impurity (see Fig. 1), respectively:

$$
\Delta_{i j}= \begin{cases}\Delta_{\delta}^{\prime} & \text { for } \quad i=0 \text { or } j=0 \\ \Delta_{\delta} & \text { for } \quad i, j \neq 0\end{cases}
$$




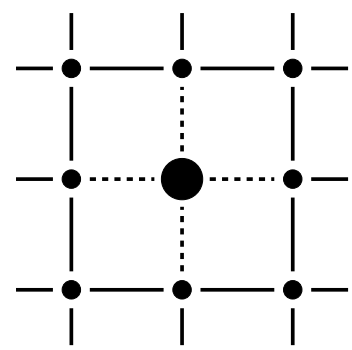

FIG. 1. Mean-field parameters $\Delta_{\delta}^{\prime}$ and $\Delta_{\delta}$ are assigned to bonds that do (dashed line) or do not (solid line) connect to the impurity site denoted by a large dot.

where $\Delta_{\delta}$ is the mean-field parameter of the impurityfree system. The two parameters $\Delta_{\delta}^{\prime}$ and $\Delta_{\delta}$ are assumed to exhibit the same phase relation, but in general their amplitudes differ. As a result, spinons scatter on the impurity bonds. To study this effect we write the spinon part of Hamiltonian (雨) as

$$
H_{\mathrm{Ni}}^{\mathrm{sp}}=H_{\mathrm{RVB}}+(1-x) \sum_{\delta \sigma}\left(\Delta_{\delta} f_{0 \sigma}^{\dagger} f_{\delta \sigma}+\text { H.c. }\right),
$$

where $H_{\mathrm{RVB}}$ represents the impurity-free system. The scattering amplitude $(1-x)$ with $x=\left|\Delta_{\delta}^{\prime} / \Delta_{\delta}\right|$ is controlled by the ratio of $J^{\prime}$ to $J$. It vanishes for $J^{\prime}=J$, and has to be treated self-consistently for $J^{\prime}<J$. Approximately, we find $x=J^{\prime} / J$. At this point, we introduce spinon propagators $g_{\lambda}^{(0)}(i \omega)=-\left\langle T_{\tau} f_{\lambda}(\tau) f_{\lambda}^{\dagger}(0)\right\rangle_{i \omega}^{0}=$ $\left(i \omega-\xi_{\lambda}\right)^{-1}$ and $g_{\lambda \lambda^{\prime}}(i \omega)=-\left\langle T_{\tau} f_{\lambda}(\tau) f_{\lambda^{\prime}}^{\dagger}(0)\right\rangle_{i \omega}$ for the pure and impurity-doped system. These can be related by a scattering matrix $T_{\lambda \lambda^{\prime}}(i \omega)$ :

$$
g_{\lambda \lambda^{\prime}}(i \omega)=g_{\lambda}^{(0)}(i \omega) \delta_{\lambda \lambda^{\prime}}+g_{\lambda}^{(0)}(i \omega) T_{\lambda \lambda^{\prime}}(i \omega) g_{\lambda^{\prime}}^{(0)}(i \omega) .
$$

A simplified notation $\lambda=(\boldsymbol{k}, \nu)$ and Matsubara frequencies $i \omega=i(2 n+1) \pi T$, where $T$ denotes temperature and $n$ integer numbers, are employed. The $T_{\lambda \lambda^{\prime}}$ matrix in Eq. (8) describes scattering of spinons on the four bonds that connect the impurity site to its nearest neighbors. We find it to be given by the expression

$$
T_{\lambda \lambda^{\prime}}(i \omega)=\frac{t_{\lambda \lambda^{\prime}}(i \omega)}{i \omega G^{(0)}(i \omega)+p^{2}},
$$

with

$$
\begin{aligned}
t_{\lambda \lambda^{\prime}}(i \omega)= & \frac{1-x}{1+x} G^{(0)}(i \omega)\left(i \omega-\xi_{\lambda}\right)\left(i \omega-\xi_{\lambda^{\prime}}\right) \\
& +\frac{x}{1+x}\left(2 i \omega-\xi_{\lambda}-\xi_{\lambda^{\prime}}\right)-i \omega .
\end{aligned}
$$

Here $G^{(0)}(i \omega)=\sum_{\lambda} g_{\lambda}^{(0)}(i \omega)=-\left(2 i \omega / D^{2}\right) \ln (D /|\omega|)$, and $p^{2}=x^{2} /\left(1-x^{2}\right)$. The important point is that in the flux phase the scattering matrix of Eq. (9) has two poles that are determined by the roots of

$$
\omega G^{(0)}\left(i \omega \rightarrow \omega+i 0^{+}\right)+p^{2}=0 .
$$
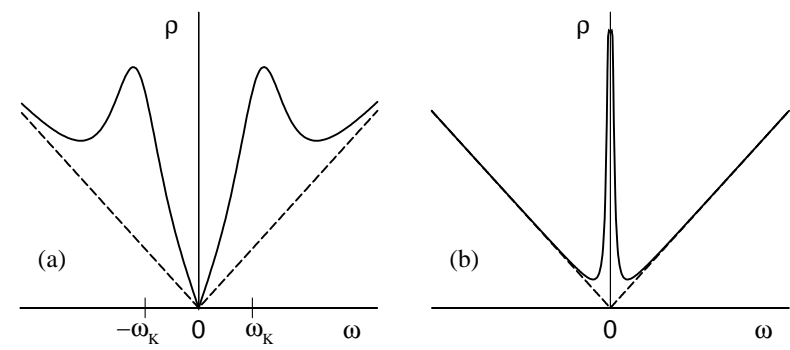

FIG. 2. Schematic plot of the spinon density of states for (a) $J^{\prime}>0$ and (b) $J^{\prime}=0$ corresponding to magnetic and nonmagnetic impurities, respectively. Solid lines represent the impurity-doped system, dashed lines the pure system. The spinon chemical potential $\mu_{s}$ lies in the center of the gap, and the $\delta$ function in (b) is artificially broadened.

One of the poles lies below the spinon chemical potential which signals the formation of a spinon bound state. This can be interpreted as follows: Due to impurity substitution, one $\mathrm{Cu}$ spin loses its RVB singlet partner. In a spin gap system in which short-range spin-singlet correlations dominate, this unpaired spin does not dissolve into the RVB ground state but rather forms a local moment distributed over $\mathrm{Cu}$ sites in the proximity of the impurity. At finite coupling $J^{\prime}$ this moment forms a local singlet with the impurity-site spinon $f_{0 \sigma}$. The characteristic binding energy $\omega_{K}$ and lifetime $\delta_{K}$ of the resulting bound state are given by the real and imaginary part of the pole, respectively. For $J^{\prime} \ll J$, one obtains

$$
\omega_{K}=\frac{\pi}{4} \frac{J^{\prime}}{\ln D / J^{\prime}}, \quad \delta_{K}=\frac{\pi}{4} \frac{\omega_{K}}{\ln D / \omega_{K}} .
$$

In the following, two different energy scales are distinguished: $\omega<\omega_{K}$ and $\omega>\omega_{K}$. These control the physics at large and short distance from the impurity as compared to $R_{K}=D / \omega_{K}$, respectively, where $R_{K}$ is measured in units of lattice spacing.

First we analyze the low-energy fixed point of the system with a magnetic impurity for which $J^{\prime}$ and hence $\omega_{K}$ are finite. It is determined by the regime $\omega<\omega_{K}$ and applies to distances $R>R_{K}$ from the impurity site. We calculate the impurity contribution $\delta \rho(\omega)$ to the density of states from the Green's function $\delta G(i \omega)=$ $\sum_{\lambda \lambda^{\prime}} g_{\lambda}^{(0)}(i \omega) T_{\lambda \lambda^{\prime}}(i \omega) g_{\lambda^{\prime}}^{(0)}(i \omega)=(\partial / \partial i \omega) \ln \left[i \omega G^{(0)}(i \omega)+\right.$ $\left.p^{2}\right]$. For $\omega \ll D$, the latter is

$$
\delta G(i \omega)=\frac{2 G^{(0)}(i \omega)}{i \omega G^{(0)}(i \omega)+p^{2}},
$$

which yields

$$
\delta \rho(\omega)=\frac{2}{\pi} \omega_{K} \delta_{K} \frac{|\omega|}{\left(\omega^{2}-\omega_{K}^{2}\right)^{2}+\left(2 \omega_{K} \delta_{K}\right)^{2}} .
$$

Figure 2 (a) schematically shows the spinon density of states $\rho^{(0)}(\omega)$ and $\rho(\omega)=\rho^{(0)}(\omega)+\delta \rho(\omega)$ for the pure and impurity-doped system. The very existence of a magnetic 
pseudogap is found to be unaffected by the presence of the impurity $-\rho^{(0)}(\omega)$ as well as $\rho(\omega)$ vanish linearly in the limit $\omega \rightarrow 0$. As a consequence, the static spin susceptihility, which is related to the spinon density of states by 10

$$
\chi(T)=\frac{1}{4 T} \int_{-\infty}^{\infty} d x \frac{\rho(x)}{\cosh ^{2}(x / 2 T)}
$$

vanishes as $\propto T$ at low temperatures. This indicates that in the low-energy limit all spins (except $\boldsymbol{S}_{\text {eff }}$ which is not part of the spinon system) participate in the formation of singlets. The spinon bound state discussed above hence partially screens the impurity spin by forming a Kondo singlet with $\boldsymbol{s}_{0}$. An effective $S=\frac{1}{2}$ impurity spin $\boldsymbol{S}_{\text {eff }}$ remains. In this underscreened Kondo problem, the spinon binding energy $\omega_{K}$ of Eq. (11) plays the role of the Kondo temperature: $T_{K}=\omega_{K}$. For temperatures $T \gg T_{K}$, the susceptibility associated with the spinon bound state is that of a free spin $\frac{1}{2}$, i.e., $\chi(T)=1 /(4 T)$; simultaniously, the original $S=1$ impurity spin is recovered. We note that the Kondo temperature exhibits an unconventional power-law dependence on the coupling parameter $J^{\prime}$, contrasting the conventional exponential behavior. This peculiarity is ascribed to the fact that the impurity spin couples to bound spinons which are predomenantly in localized rather than bandlike states. Finally, we shortly discuss how the presence of a Kondo singlet affects the properties of the spinon system at $T \ll T_{K}$. Although the impurity does not fill the magnetic pseudogap, it nevertheless renormalizes its slope. The leading term in a low-energy expansion of Eq. (13) is related to the density of states of the pure system by

$$
\delta \rho(\omega)=\frac{1}{p^{2}} \rho^{(0)}(\omega),
$$

valid for $J^{\prime} \ll J$. At low energy and large distance from the impurity, the spinon system hence behaves qualitatively as in the impurity-free case.

To finish the discussion of magnetic moments, we turn to the case of a nonmagnetic impurity. The relevant physics is modelled by decoupling the spinon sector from the impurity site, setting $J^{\prime}=0$, and by discarding contributions stemming from the impurity spin which is now free. Since $\omega_{K}$ consequently vanishes, one is always in the regime $\omega>\omega_{K}$. A Kondo singlet cannot form even in the zero-energy limit as the impurity carries no inherent spin. The spinon bound state induced by the impurity lies at the spinon chemical potential in the center of the pseudogap [see Fig. 2(b)]:

$$
\delta \rho(\omega)=\delta(\omega) .
$$

This is associated with the magnetic susceptibility $1 /(4 T)$ of a free spin $\frac{1}{2}$ which holds down to zero temperature. We note that the impurity-induced moment is broadly distributed over planar $\mathrm{Cu}$ sites on sublattice $B$ that does not contain the impurity site, its density falling off as $R^{-2}$ with distance from the impurity.

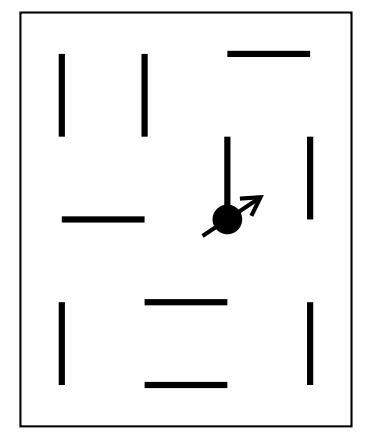

(a)

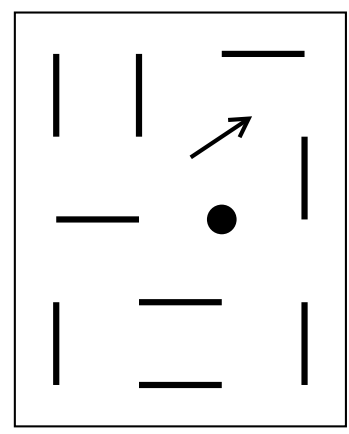

(b)
FIG. 3. "Snapshot" of the low-energy fixed point of a RVB liquid state with (a) the $S=1$ magnetic impurity and (b) the nonmagnetic impurity denoted by a dot. In the former case, the impurity spin is partially screened by moments induced on $\mathrm{Cu}$ sites. Effectively, a local impurity spin $\frac{1}{2}$ and a "healed" spin liquid results. In the latter case, the impurity induces a broadly distributed moment that resides on $\mathrm{Cu}$ sites in the proximity of the impurity.

To summarize, magnetic Ni and nonmagnetic Zn impurities are both associated with $S=\frac{1}{2}$ magnetic moments. These are, however, of very different natures (see Fig. 3): In the former case, the spinon bound state partially screens the original $S=1$ impurity spin. One is left with an effective impurity spin $\frac{1}{2}$ ferromagnetically coupled to an ensemble of inherent spinons that, in the absence of a magnetic field, behaves qualitatively the same as an impurity-free system. In the latter case, the moment is carried by the spinon bound state itself, and is broadly distributed over $\mathrm{Cu}$ sites.

\section{SPIN POLARIZATION}

The effective impurity moments discussed in Sec. III can be polarized by applying an external magnetic field. In this section we analyze the incidental local response of planar $\mathrm{Cu}$ spins. In the case of a magnetic impurity, the applied field acts on a localized impurity spin $\frac{1}{2}$ ferromagnetically coupled to the spin liquid. $\mathrm{Cu}$ spins respond via a RKKY-type interaction. In the case of a nonmagnetic impurity, the moment itself resides on $\mathrm{Cu}$ sites. Applying a magnetic field therefore directly polarizes the $\mathrm{Cu}$ spins.

We first discuss the situation of a magnetic impurity. The static polarizability is defined by $K_{\mathrm{Ni}}(T, \boldsymbol{R})=$ $\left\langle T_{\tau} s_{\boldsymbol{R}}^{z}(\tau) S_{\text {eff }}^{z}(0)\right\rangle_{\omega=0}$, where $s_{\boldsymbol{R}}^{z}$ and $S_{\text {eff }}^{z}$ denote the $z$ components of a given $\mathrm{Cu}$ spin at site $\boldsymbol{R}$ and of the effective impurity spin, respectively. It is expressed in terms of Green's functions as (see Fig. (1)

$$
K_{\mathrm{Ni}}(T, \boldsymbol{R})=-T^{2} \sum_{\varepsilon, \varepsilon^{\prime}} \Pi_{d}\left(i \varepsilon^{\prime}\right) J_{c}\left(i \varepsilon-i \varepsilon^{\prime}\right) \Pi_{f}(i \varepsilon, \boldsymbol{R})
$$

with particle-hole convolution functions 


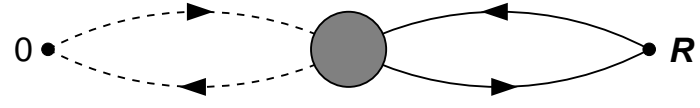

FIG. 4. Diagrammatic representation of the static polarization $K_{\mathrm{Ni}}(T, \boldsymbol{R})$ of a $\mathrm{Cu}$ spin at site $\boldsymbol{R}$ due to RKKY coupling to the localized impurity moment. Dashed and solid ovals represent particle-hole convolution functions $\Pi_{d}\left(i \varepsilon^{\prime}\right)$ for the local moment and $\Pi_{f}(i \varepsilon, \boldsymbol{R})$ for itinerant spinons, respectively. The effective coupling is described by the vertex function $J_{c}\left(i \varepsilon-i \varepsilon^{\prime}\right)$ denoted by a circle.

$$
\begin{aligned}
\Pi_{d}(i \varepsilon) & =D^{2}(i \varepsilon), \\
\Pi_{f}(i \varepsilon, \boldsymbol{R}) & =G(i \varepsilon,-\boldsymbol{R}) G(i \varepsilon, \boldsymbol{R}) .
\end{aligned}
$$

Here the impurity Green's function is $D(i \omega)=$ $-\left\langle T_{\tau} d_{\sigma}(\tau) d_{\sigma}^{\dagger}(0)\right\rangle_{i \omega}=1 /(i \omega)$, and the intersite spinon Green's function $G(i \omega, \boldsymbol{R})=-\left\langle T_{\tau} f_{0 \sigma}(\tau) f_{\boldsymbol{R} \sigma}^{\dagger}(0)\right\rangle_{i \omega}$. Operators $d$ and $f$ act on separated sectors of the Hilbert space. At site $\boldsymbol{R}=0$, however, $f$ spinons are polarized by the local spin $\boldsymbol{S}_{\text {eff }}$ due to the ferromagnetic interaction of bare strength $J_{c}$. This coupling is accounted for by the vertex function $J_{c}(i \omega)$. Employing a ladder approximation it is

$$
J_{c}(i \omega)=\frac{J_{c}}{1+J_{c} \Pi_{c}^{i \omega}}=\frac{1}{\Pi_{c}^{i \omega}}
$$

with

$$
\Pi_{c}^{i \omega}=-T \sum_{\varepsilon} D(i \varepsilon+i \omega) G(i \varepsilon, \boldsymbol{R}=0) .
$$

The second equality in Eq. (18) holds due to $J_{c}$ being infinitely large. Replacing the vertex function by its zerofrequency limit, $J(i \omega) \rightarrow J(0)$, the polarizability of Eq. (17) can be factorized. Within this approximation, which is valid at low temperatures, one obtains

$$
K_{\mathrm{Ni}}(T, \boldsymbol{R})=\chi_{\mathrm{eff}}(T) J_{c}(0) \chi_{\mathrm{pl}}(T, \boldsymbol{R}) .
$$

The polarizability has thus been decomposed into the magnetic susceptibility of the effectively free $\frac{1}{2}$ impurity spin, $\chi_{\mathrm{eff}}(T)=1 /(4 T)$, the nonlocal magnetic susceptibility of $\mathrm{CuO}_{2}$ planes, $\chi_{\mathrm{pl}}(T, \boldsymbol{R})$, and an effective coupling parameter $J_{c}(0)$. The susceptibilities are defined as $\chi_{\mathrm{eff}}(T)=\left\langle T_{\tau} S_{\mathrm{eff}}^{z}(\tau) S_{\mathrm{eff}}^{z}(0)\right\rangle_{\omega=0}=-T \sum_{\varepsilon} \Pi_{d}(i \varepsilon)$ and $\chi_{\mathrm{pl}}(T, \boldsymbol{R})=\left\langle T_{\tau} s_{\boldsymbol{R}}^{z}(\tau) s_{0}^{z}(0)\right\rangle_{\omega=0}=-T \sum_{\varepsilon} \Pi_{f}(i \varepsilon, \boldsymbol{R})$.

To further analyze the polarizability in Eq. (19), $J_{c}(0)$ and $\chi_{\mathrm{pl}}(\boldsymbol{R})$ have to be evaluated. This requires the onsite and intersite spinon Green's functions

$$
G(i \omega, \boldsymbol{R})=\left\{\begin{array}{l}
\left(\frac{p}{x}\right)^{2} \frac{G^{(0)}(i \omega)}{i \omega G^{(0)}(i \omega)+p^{2}} \text { for } R=0 \\
\frac{1}{x} G^{(0)}(i \omega, \boldsymbol{R}) \text { for } \quad R>R_{K},
\end{array}\right.
$$

where $G^{(0)}(i \omega, \boldsymbol{R})$ is defined for the impurity-free system

$$
G^{(0)}(i \omega, \boldsymbol{R})=-\frac{2 i|\omega|}{D^{2}} \varphi(\boldsymbol{R}) K_{1}\left(\frac{R|\omega|}{D}\right),
$$

with a modified Bessel function of the second kind, $K_{\nu}(x)$. Equation (21) holds for sites on sublattice $B$; contributions from sublattice $A$ containing the impurity are found to be negligible. The angular dependence is determined by the phase factor

$$
\varphi(\boldsymbol{R})=\frac{1}{2}\left(\tilde{R}^{+} e^{i \pi R^{+} / 2}+\tilde{R}^{-} e^{i \pi R^{-} / 2}\right)
$$

with $R^{ \pm}=R_{x} \pm R_{y}$ and $\tilde{R}^{ \pm}=\left(R_{x} \pm i R_{y}\right) / R$. We are now in the position to calculate the effective coupling parameter from the zero-frequency limit of Eq. (18),

$$
J_{c}(0)=\left\{\begin{array}{l}
D \quad \text { for } \quad J^{\prime}=J \\
2 \omega_{K} \text { for } \quad J^{\prime} \ll J,
\end{array}\right.
$$

and the nonlocal spin susceptibility of $\mathrm{CuO}_{2}$ planes in the presence of the impurity,

$$
\chi_{\mathrm{pl}}(\boldsymbol{R})=-\frac{3}{4 \pi} \frac{1}{J x^{2}} \frac{\Phi(\boldsymbol{R})}{R^{3}},
$$

the latter being valid for $\boldsymbol{R} \in B$ with $R>R_{K}$. The phase factor in Eq. 24 is defined by $\Phi(\boldsymbol{R})=|\varphi(\boldsymbol{R})|^{2}$. Finally, combining these results, we obtain

$$
K_{\mathrm{Ni}}(T, \boldsymbol{R})=-\frac{3}{16 \pi} \frac{J_{c}(0)}{J x^{2}} \frac{\Phi(\boldsymbol{R})}{R^{3}} \frac{1}{T},
$$

which describes the polarizability of a $\mathrm{Cu}$ spin at site $\boldsymbol{R} \in B$ responding to a magnetic field that acts on the effective impurity spin $\boldsymbol{S}_{\text {eff }}$; contributions from sublattice $A$ are found to be small. We note that the $T^{-1}$ Curie behavior displayed by Eq. (25) stems solely from the susceptibility $\chi_{\mathrm{eff}}(T)$ of the effective impurity spin. Within the present mean-field treatment, the planar susceptibility is independent of temperature: $\chi_{\mathrm{pl}}(T, \boldsymbol{R})=\chi_{\mathrm{pl}}(\boldsymbol{R})$.

We now briefly review the result for a nonmagnetic impurity which was derived in Ref. 6. Here, the $\mathrm{Cu}$ spins carry the impurity-induced moment, and can therefore be directly polarized by the magnetic field. The polarizability is given by the local susceptibility of the impurity-induced moment, $K_{\mathrm{Zn}}(T, \boldsymbol{R})=\delta \chi_{\mathrm{pl}}(T, \boldsymbol{R})=$ $\sum_{\boldsymbol{R}^{\prime}}\left(\left\langle T_{\tau} s_{\boldsymbol{R}}^{z}(\tau) s_{\boldsymbol{R}^{\prime}}^{z}(0)\right\rangle_{\omega=0}-\left\langle T_{\tau} s_{\boldsymbol{R}}^{z}(\tau) s_{\boldsymbol{R}^{\prime}}^{z}(0)\right\rangle_{\omega=0}^{0}\right)$, yielding

$$
K_{\mathrm{Zn}}(T, \boldsymbol{R})=\frac{1}{2 \pi} \frac{\Phi(\boldsymbol{R})}{R^{2}} \frac{1}{T \ln D / T} .
$$

Equation (26) is valid for $\boldsymbol{R} \in B$, while contributions from $\boldsymbol{R} \in A$ are again negligible. The polarizability is found to decay slowly as $R^{-2}$ with distance from the impurity which compares to a $R^{-3}$ behavior in the case of $\mathrm{Ni}$, reflecting the delocalized nature of the moment induced by a Zn impurity. Further, a logarithmic correction to the Curie-like temperature behavior is to be marked. 
In deriving Eqs. (25) and (26) for the polarizability of $\mathrm{Cu}$ spins, we have, up to this point, built upon RVB mean-field theory. This picture accounts well for the spin liquid features of underdoped cuprates including the presence of a magnetic pseudogap. Its strength lies on the description of long-range properties controlled by lowenergy excitations. The mean-field treatment does, however, severely underestimate local AF correlations which reflect the proximity of a critical instability towards $\mathrm{AF}$ spin ordering. As a consequence, the above expressions contain no reference to the AF correlation length which was suggested to introduce a temperature dependence beyond the Curie behavior of free moments 3 Furthermore, mean-field theory yields a polarizability of $\mathrm{Cu}$ spins on one sublattice only, undervaluing the staggered magnetization of spins on the opposite suplattice. This is in disaccord with NMR measurements that yield no overall shift of the ${ }^{17} \mathrm{O}$ line, as would be expected from the polarization ff only one sublattice as well as with numerical studies.11

To compensate for these deficiencies of the mean-field treatment, we simulate the closeness of the spin system towards an antiferromagnetically ordered state by performing a random-phase approximation (RPA) in the magnetic susceptibility. In the momentum representation, the susceptibility of planar $\mathrm{Cu}$ spins then becomes

$$
\chi_{\mathrm{pl}}^{\mathrm{RPA}}(T, \boldsymbol{q})=\chi_{\mathrm{pl}}(\boldsymbol{q}) S(T, \boldsymbol{q})
$$

with the Stoner enhancement factor

$$
S(T, \boldsymbol{q})=\frac{1}{1+J_{\boldsymbol{q}} \chi_{\mathrm{pl}}(T, \boldsymbol{q})},
$$

where $J_{\boldsymbol{q}}=2 J\left(\cos q_{x}+\cos q_{y}\right)$. We closely follow the theory of a nearly AF Fermi liquid,2 2 which maps Eq. (27) onto a phenomenological expression involving the AF correlation length $\xi(T)$. Within this picture, $\chi_{\mathrm{pl}}^{\mathrm{RPA}}(T, \boldsymbol{q})$ is assumed to be controlled solely by the momentum region close to the $\mathrm{AF}$ wave vector $\boldsymbol{Q}=$ $(\pi, \pi)$. However, we do take a slightly different point of view in this respect: The momentum dependence of the bare susceptibility $\chi_{\mathrm{pl}}(\boldsymbol{q})$ in Eq. (27), which describes the long-range characteristics of spin correlations in the presence of a magnetic pseudogap, is explicitly kept. Only the scaling function $S(T, \boldsymbol{q})$, which controls shortrange AF correlations, is expanded around $\boldsymbol{Q}=(\pi, \pi)$. Identifying $J \chi_{\mathrm{pl}}(T, \boldsymbol{Q}) /\left[1-4 J \chi_{\mathrm{pl}}(T, \boldsymbol{Q})\right]=\xi^{2}(T)$ and $1 /\left[J \chi_{\mathrm{pl}}(T, \boldsymbol{Q})\right]=\alpha$, Eq. (28) can be written in phenomenological form

$$
S(T, \boldsymbol{q})=\frac{\alpha \xi^{2}(T)}{1+(\boldsymbol{q}-\boldsymbol{Q})^{2} \xi^{2}(T)},
$$

where $\alpha \approx 1$ on a mean-field level. We note that the explicit form of $\xi(T)$ lies beyond the accessibility of a mean-field treatment, and has to be chosen according to general physical considerations.

Turning back to real space, the nonlocal susceptibility is

$$
\chi_{\mathrm{pl}}^{\mathrm{RPA}}(T, \boldsymbol{R})=\sum_{\boldsymbol{R}^{\prime} \in B} \chi_{\mathrm{pl}}\left(\boldsymbol{R}^{\prime}\right) S\left(T, \boldsymbol{R}-\boldsymbol{R}^{\prime}\right) .
$$

For distances $R \gg \xi(T)$, it can be approximated by $\chi_{\mathrm{pl}}^{\mathrm{RPA}}(T, \boldsymbol{R})=\chi_{\mathrm{pl}}(\boldsymbol{R}) \xi^{2}(T) / 2$ with interpolation formula for the $A$ sublattice $\chi_{\mathrm{pl}}(\boldsymbol{R} \in A)=-(1 / z) \sum_{\boldsymbol{\delta}} \chi_{\mathrm{pl}}(\boldsymbol{R}+\boldsymbol{\delta})$. Analogous expressions are obtained for the local susceptibility $\delta \chi_{\mathrm{pl}}(T, \boldsymbol{R})$ induced by a nonmagnetic impurity. Combining these results with Eqs. (25) and (26) and performing an angular average over phase factors $\Phi(\boldsymbol{R})$, one finally arrives at the following expressions for the polarizability of $\mathrm{Cu}$ spins in the impurity-doped system:

$$
\begin{aligned}
& K_{\mathrm{Ni}}(T, \boldsymbol{R})=\cos (\boldsymbol{Q R}) \frac{3}{64 \pi} \frac{J_{c}(0)}{J x^{2}} \frac{1}{R^{3}} \frac{\xi^{2}(T)}{T} \\
& K_{\mathrm{Zn}}(T, \boldsymbol{R})=-\cos (\boldsymbol{Q R}) \frac{1}{8 \pi} \frac{1}{R^{2}} \frac{\xi^{2}(T)}{T \ln D / T}
\end{aligned}
$$

These equations now hold for both sublattices, $\boldsymbol{R} \in$ $\{A, B\}$, the staggered nature of spin correlations being manifested in the alternating sign implied by $\cos (\boldsymbol{Q R})$. Further, the dependence upon the AF correlation length $\xi(T)$ is now explicitly accounted for.

\section{NMR LINE BROADENING}

The impurity-induced polarization of $\mathrm{Cu}$ spins in a magnetic field affects the energy levels of nuclear spins via supertransferred hyperfine interaction. The coupling of a given nuclear spin $\boldsymbol{I}$ to electron spins $\boldsymbol{s}_{i}$ on close by $\mathrm{Cu}$ sites is described by

$$
H_{\mathrm{hf}}=\gamma_{n} \gamma_{e} C_{\mathrm{hf}} \sum_{i} \boldsymbol{s}_{i} \boldsymbol{I},
$$

where $\gamma_{n}$ and $\gamma_{e}$ denote the nuclear and electron gyromagnetic ratios, respectively, and $C_{\mathrm{hf}}$ is the supertransferred hyperfine coupling constant. In the following, we restrict ourselves to NMR measurements on ${ }^{17} \mathrm{O}$ nuclei $\left(I=\frac{5}{2}\right)$. On a mean-field level, $\boldsymbol{s}_{i}$ can be replaced by its average value $\left\langle\boldsymbol{s}_{i}\right\rangle=K\left(T, \boldsymbol{R}_{i}\right) \boldsymbol{H}_{0}$ with external magnetic field $\boldsymbol{H}_{0}$ and polarizability $K\left(T, \boldsymbol{R}_{i}\right)$ given by either one of Eqs. (31) and (32) for the two types of impurities. Since each ${ }^{17} \mathrm{O}$ nucleus lies symmetrically in between two $\mathrm{Cu}$ sites that belong to different sublattices with spins polarized in opposite directions, the impurity-induced energy shift partially cancels (see Fig. 5). At large enough distance from the impurity, the shift is then effectively determined by the spatial derivative of the polarizability,

$$
\omega(\boldsymbol{R})=\kappa \frac{\partial|K(T, \boldsymbol{R})|}{\partial R} \cos \phi,
$$

where $\phi$ denotes the angle enclosed by $\boldsymbol{R}$ and the $x$ or $y$ axis and $\kappa=\gamma_{n} \gamma_{e} C_{\mathrm{hf}} H_{0}$.

In a system with randomly distributed impurities of concentration $c$, the superposition of energy shifts induced by different impurities leads to a broadening of the 


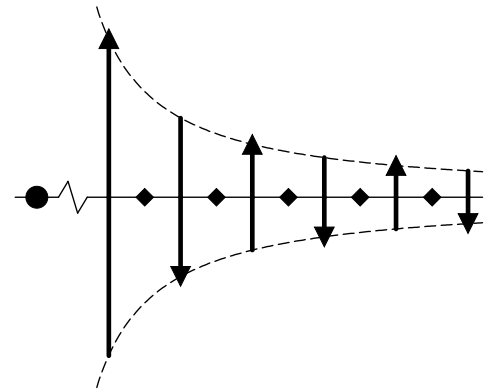

FIG. 5. Schematic cut through a $\mathrm{CuO}_{2}$ plane, showing the position of $\mathrm{O}$ ions (diamonds) placed in between successive $\mathrm{Cu}$ sites with staggered spin polarization (arrows). The magnitude of the polarization falls off with distance from the impurity (circle) as $R^{-3}$ in the case of $\mathrm{Ni}$, and as $R^{-2}$ in the case of $\mathrm{Zn}$.

NMR line. We calculate the line shape that follows from Eq. (34), employing the formalism of Ref. 13. The line shape function $g(\nu)$ is defined as the Fourier transform of the characteristic or free-induction function

$$
f(t)=\exp \left[-c \sum_{\boldsymbol{R}}\left(1-e^{i \omega(\boldsymbol{R}) t}\right)\right] .
$$

Integrating over lattice sites, for $\mathrm{Ni}$ and $\mathrm{Zn}$, respectively, one obtains

$$
\ln f(t)=\left\{\begin{array}{l}
-\Lambda_{\mathrm{Ni}}|t|^{1 / 2} \\
-\Lambda_{\mathrm{Zn}}|t|^{2 / 3}
\end{array}\right.
$$

with

$$
\begin{aligned}
& \Lambda_{\mathrm{Ni}}=\frac{2 \sqrt{6} \pi \Gamma(3 / 4)}{\Gamma(1 / 4)}\left[\kappa \frac{3}{64 \pi} \frac{J_{c}(0)}{J x^{2}} \frac{\xi^{2}(T)}{T}\right]^{1 / 2} c \\
& \Lambda_{\mathrm{Zn}}=\frac{2 \sqrt{3} \pi^{2}}{\Gamma^{2}(1 / 3)}\left[\kappa \frac{1}{8 \pi} \frac{\xi^{2}(T)}{T \ln D / T}\right]^{2 / 3} c .
\end{aligned}
$$

Figure 6 shows the different line shapes induced by Ni and $\mathrm{Zn}$ impurities as obtained by performing a Fourier transformation on $f(t)$. Comparing to the Lorentzian shape that results from $\ln f(t) \propto-|t|$ in conventional RKKY theory, one finds a marked difference in both shape and width. Using the numerical values shown in the inset of Fig. 6, we finally arrive at the following expressions for the full linewidth induced by magnetic and nonmagnetic impurities:

$$
\begin{aligned}
& \Delta \nu_{\mathrm{Ni}}=2 \times 0.22\left(\Lambda_{\mathrm{Ni}}\right)^{2}, \\
& \Delta \nu_{\mathrm{Zn}}=2 \times 0.51\left(\Lambda_{\mathrm{Zn}}\right)^{2 / 3} .
\end{aligned}
$$

\section{COMPARISON WITH EXPERIMENT}

In this section, we compare the impurity-induced ${ }^{17} \mathrm{O}$ NMR line broadening as described by Eqs. (37) and (38)

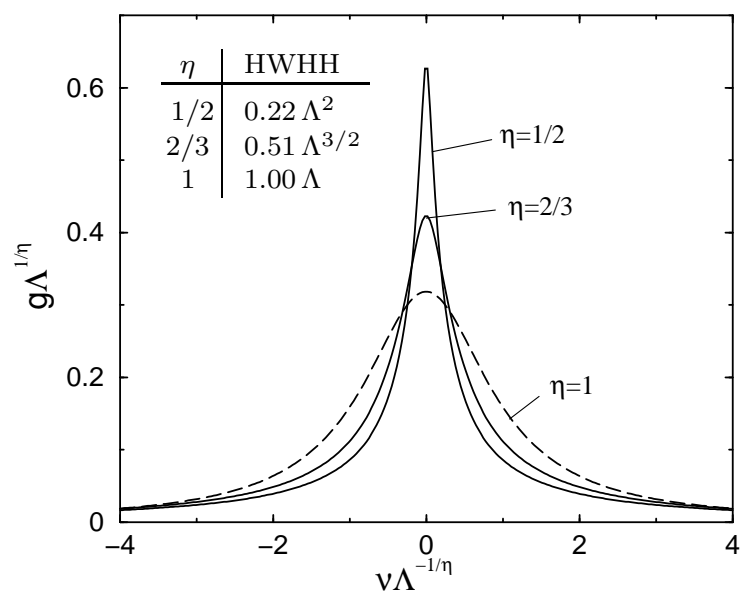

FIG. 6. Line-shape function $g(\nu)$ obtained by performing a Fourier transformation on the characteristic function $f(t)=\exp \left[-\Lambda|t|^{\eta}\right]$ with $\eta=\frac{1}{2}$ for $\mathrm{Ni}$ and $\eta=\frac{2}{3}$ for Zn. For comparison, the Lorentzian line shape of conventional RKKY theory with $\eta=1$ is indicated by a dashed line. Numerical values for the half width at half height $(\mathrm{HWHH})$ are given in the inset.

with experimental data of Bobroff et al. $\mathrm{YBa}_{2}\left(\mathrm{Cu}_{1-x} M_{x}\right)_{3} \mathrm{O}_{6.6}$ with $M=\mathrm{Zn}$ or $\mathrm{Ni}$.

The following constants are chosen: The superexchange parameters are specified by $J=0.13 \mathrm{eV}$ for $\mathrm{Cu}-\mathrm{Cu}$ interaction and $J^{\prime}=J / 2$ for $\mathrm{Cu}-\mathrm{Ni} .14$ A selfconsistent treatment yields a scattering amplitude $(1-$ $x)=0.5$, where $\Delta=\frac{1}{4}$ has been used. The Kondo temperature is obtained by numerically solving Eq. (10) which gives $T_{K}=560 \mathrm{~K}$. Below this temperature, the $\mathrm{Ni}$ spin is partially screened and behaves as a spin $\frac{1}{2}$ ferromagnetically coupled to the $\mathrm{CuO}_{2}$ plane. The effective coupling constant of this interaction given by Eq. (23) is $J_{c}(0)=0.1 \mathrm{eV}$. The hyperfine coupling constant between ${ }^{17} \mathrm{O}$ nuclear and $\mathrm{Cu}$ electron spins is $C_{\mathrm{hf}}=3.3 \mathrm{~T} / \mu_{B}$. The magnetic-field strength used in the experiment is $H_{0}=7.5 \mathrm{~T}$, and the concentration of $\mathrm{Ni}$ and $\mathrm{Zn}$ impurities is $1 \%$. The effective impurity concentration within $\mathrm{CuO}_{2}$ planes, which is larger by a factor of $\frac{3}{2}$, is finally $c=1.5 \%$.

Next, an expression for the AF correlation length $\xi(T)$ has to be specified. It is argued in Ref. 15 that below a critical temperature $T_{\text {cr }}$ specified by $\xi\left(\bar{T}_{\text {cr }}\right) \approx 2$, the correlation length assumes the form

$$
\xi(T)=\frac{1}{a+b T},
$$

where $a$ and $b$ are fitting constants of the theory. Saturation of $\xi(T)$ at low temperatures is neglected here.

Figure 6 shows the impurity-induced line broadening $\Delta \nu_{\text {imp }}$ scaled with temperature. The curves are fitted to the experimental data by setting $a=0.07$ and $b=$ 0.0007 , which correspond to an AF correlation length of $\xi=4.8$ in units of lattice spacings at $T=200 \mathrm{~K}$. This compares well to $\xi=5.9$ obtained in Ref. 16. No further 


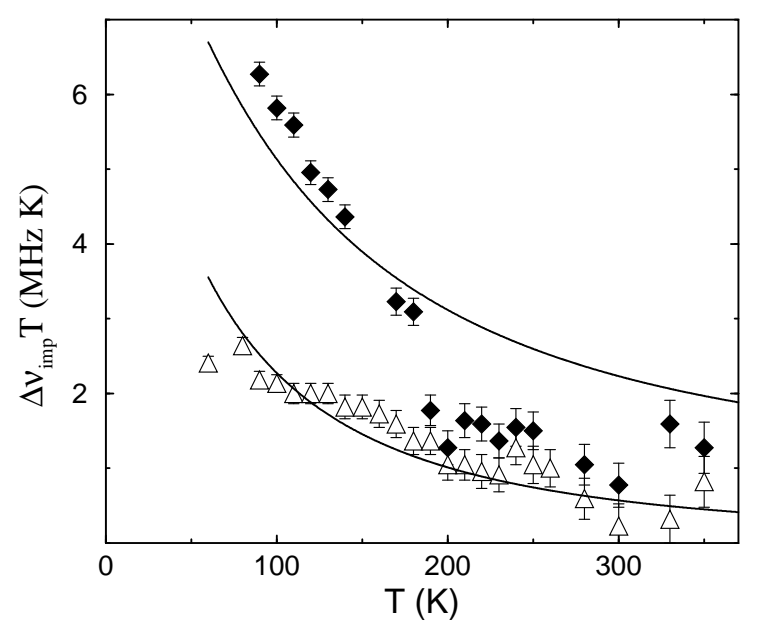

FIG. 7. Impurity-induced NMR line broadening $\Delta \nu_{\mathrm{imp}}$ multiplied by temperature. The theoretical result is indicated by solid lines fitted to experimental ${ }^{17} \mathrm{O}$ data for $1 \% \mathrm{Ni}$-doped (triangles) and $1 \% \mathrm{Zn}$-doped (diamonds) $\mathrm{YBa}_{2}\left(\mathrm{Cu}_{1-x} M_{x}\right)_{3} \mathrm{O}_{6.6}, M=\mathrm{Ni}$ or $\mathrm{Zn}$.

fitting parameters are needed.

The theory correctly accounts for the peculiar experimental observation of $\mathrm{Zn}$ having a more pronounced effect on the NMR signal than $\mathrm{Ni}$ - this seems to be in contradiction to SQUID measurements on the macroscopic susceptibility. 1 We are able to ascribe this behavior to the different spatial dependence of the polarizability: $K(T, \boldsymbol{R})$ decays as $R^{-3}$ in the case of $\mathrm{Ni}$, but only as $R^{-2}$ in the case of $\mathrm{Zn}$. Averaging over all impurity site, this leads to an enhanced line-broadening effect of Zn (see Fig. 6). Our theory further correctly describes the anomalous non-Curie temperature dependence exhibited by the NMR linewidth - this seems to be in disaccord with an almost perfect $T^{-1}$ behavior exhibited by the macroscopic susceptibility. 1 One can resolve this disagreement by assuming a temperature dependence of the AF correlation length $\xi(T)$ which enters the polarizability of $\mathrm{Cu}$ spins. Good agreement with experiment is obtained by employing $\xi(T)$ of the form given in Eq. (39).

\section{CONCLUSION}

In summary, we have studied local moments induced in underdoped cuprates by doping with magnetic $(S=1)$ $\mathrm{Ni}$ and nonmagnetic $\mathrm{Zn}$ impurities. In the presence of a spin gap, both types of impurities are associated with $S=\frac{1}{2}$ magnetic moments in the $\mathrm{CuO}_{2}$ planes. These are, however, of very different natures. $\mathrm{Ni}$ as well as $\mathrm{Zn}$ disturb the spin liquid formed by planar $\mathrm{Cu}$ spins, resulting in a magnetic moment residing on $\mathrm{Cu}$ sites in the proximity of the impurity. In the case of $\mathrm{Ni}$, this moment partially shields the impurity spin below a critical temperature $T_{K}$ in what resembles an underscreened Kondo model; an effective impurity spin $\frac{1}{2}$ results. Since predominantly localized rather than bandlike states are in- volved in the screening of the impurity spin, the Kondo temperature exhibits an unconventional power-law dependence on the coupling constant. In the case of $\mathrm{Zn}$, on the other hand, one deals with a $S=\frac{1}{2}$ moment broadly distributed over $\mathrm{Cu}$ sites. We have further investigated the RKKY-type response of $\mathrm{Cu}$ spins in a magnetic field. The spin polarization is found to decay as $R^{-3}$ with distance from a Ni impurity, but only as $R^{-2}$ in the case of $\mathrm{Zn}$. This different behavior reflects the delocalized character of Zn moments, and explains why Zn has a stronger impact on the NMR linewidth than Ni. Further, accounting for the presence of temperature-dependent $\mathrm{AF}$ correlations in underdoped cuprates, we can successfully describe the non-Curie behavior of the impurity effect on the NMR linewidth. In general, it can be concluded that the anomalous impurity properties of underdoped cuprates are a clear manifestation of the peculiar mixture of spin-singlet and antiferromagnetic correlations present in these compounds.

* Permanent address: Kazan Physico Technical Institute, 420029 Kazan, Russia.

${ }^{1}$ P. Mendels, H. Alloul, G. Collin, N. Blanchard, J.-F. Marucco, and J. Bobroff, Physica C 235-240, 1595 (1994).

${ }^{2}$ J. Bobroff, H. Alloul, Y. Yoshinari, A. Keren, P. Mendels, N. Blanchard, G. Collin, and J.-F. Marucco, Phys. Rev. Lett. 79, 2117 (1997); Physica C 282-287, 1389 (1997).

${ }^{3}$ D. K. Morr, J. Schmalian, R. Stern, and C. P. Slichter, Phys. Rev. Lett. 80, 3662 (1998); Phys. Rev. B 58, 11193 (1998).

${ }^{4}$ P. W. Anderson, Science 235, 1196 (1987).

${ }^{5}$ I. Affleck and J. Marston, Phys. Rev. B 37, 3774 (1988); Y. Suzumura, Y. Hasegawa, and H. Fukuyama, J. Phys. Soc. Jpn. 57, 2768 (1988); G. Kotliar, Phys. Rev. B 37, 3664 (1988).

${ }^{6}$ G. Khaliullin, R. Kilian, S. Krivenko, and P. Fulde, Phys. Rev. B 56, 11882 (1997); Physica C 282-287, 1749 (1997).

${ }^{7}$ N. Nagaosa and T. K. Ng, Phys. Rev. B 51, 15588 (1995).

${ }^{8}$ N. Nagaosa and P. A. Lee, Phys. Rev. Lett. 79, 3755 (1997).

${ }^{9}$ C. Pépin and P. A. Lee, Phys. Rev. Lett. 81, 2779 (1998).

10 Susceptibilities are corrected by a factor of 2 to compensate for an underestimation in the present mean-field treatment. The use of a Majorana fermion representation as in Ref. 6 makes this correction dispensable.

${ }^{11}$ G. B. Martins, M. Laukamp, J. Riera, and E. Dagotto, Phys. Rev. Lett. 78, 3563 (1997); M. Laukamp, G. B. Martins, C. Gazza, A. L. Malvezzi, E. Dagotto, P. Hansen, A. C. López, and J. Riera, Phys. Rev. B 57, 10755 (1998).

${ }^{12}$ P. Monthoux and D. Pines, Phys. Rev. B 50, 16015 (1994).

${ }^{13}$ R. Walstedt and L. R. Walker, Phys. Rev. B 9, 4857 (1974).

$14 \mathrm{The} \mathrm{Ni}^{2+}$ ion with spin 1 has two holes on the 3D shell in $d_{x^{2}-y^{2}}$ and $d_{3 z^{2}-r^{2}}$ orbitals. Since superexchange is mainly contributed to by the $d_{x^{2}-y^{2}}$ orbital, one obtains $J^{\prime} / J=$ 
$1 / 2$.

15 B. P. Stojković and D. Pines, Phys. Rev. B 56, 11931 (1997).

${ }^{16}$ V. Barzykin and D. Pines, Phys. Rev. B 52, 13585 (1995). 